\title{
SpaceCAPS: Automated Mission Planning for the TechSat 21 Formation- Flying Cluster Experiment
}

\author{
John L. Mohammed \\ Stottler Henke Associates, Inc. \\ 1660 South Amphlett Boulevard, Suite 350 \\ San Mateo, California 94402 \\ mohammed@shai.com
}

\begin{abstract}
This paper describes a project concerned with the development of a mission planning system to provide automated planning assistance for the Air Force TechSat 21 formation-flying satellite cluster experiment: Spacecraft Cluster Automatic Planner/Scheduler (SpaceCAPS). The project's focus is on planning and scheduling for payload management, but the system serves as a central planning system for planning and scheduling of all spacecraft functions. The paper describes some of the planning and scheduling requirements for cluster payload management, and describes a system for optimization of the mission plan for the TechSat 21 space-based radar surveillance system.
\end{abstract}

\section{Introduction}

A satellite cluster is a group of satellites that fly within very close range of each other (e.g., $250 \mathrm{~m}-5 \mathrm{~km}$ ). These satellites coordinate their activities, so that they can use sparse array interferometry and synthetic aperture techniques to simulate a single, very large satellite. The cluster operates as a "virtual" satellite with a very large effective aperture, without the need for the heavy infrastructure that would be required to have a monolithic satellite with the equivalent aperture. The cluster approach has many advantages over a single large satellite:

- Each spacecraft is smaller, lighter, simpler, and simpler to manufacture;

- Economies of scale enable a cluster of many satellites to be less expensive to manufacture than a single satellite;

- The cluster can adapt to the failure of any individual satellites, and failed satellites can be incrementally replaced;

- The cluster can reconfigure the orbits of the satellites in the cluster to optimize for different missions.

Copyright $($ C 2002, American Association for Artificial Intelligence (www.aaai.org). All rights reserved.
A constellation of clusters would enable whole-earth coverage from low earth orbit and/or continuous coverage for specific theatres. However, clusters and constellations present a significant challenge to current methods for the management of space-based assets. Current practices are too labor-intensive and would not scale well to the large numbers of satellites that would have to be managed. Automation will have to play a much larger role in planning and operations, and tools for automation will have to be knowledgeable about the unique characteristics of the formation-flying satellite cluster. The number of satellites to be managed, and the wider range of parameters that can be optimized demand new tools for planning, scheduling and optimization.

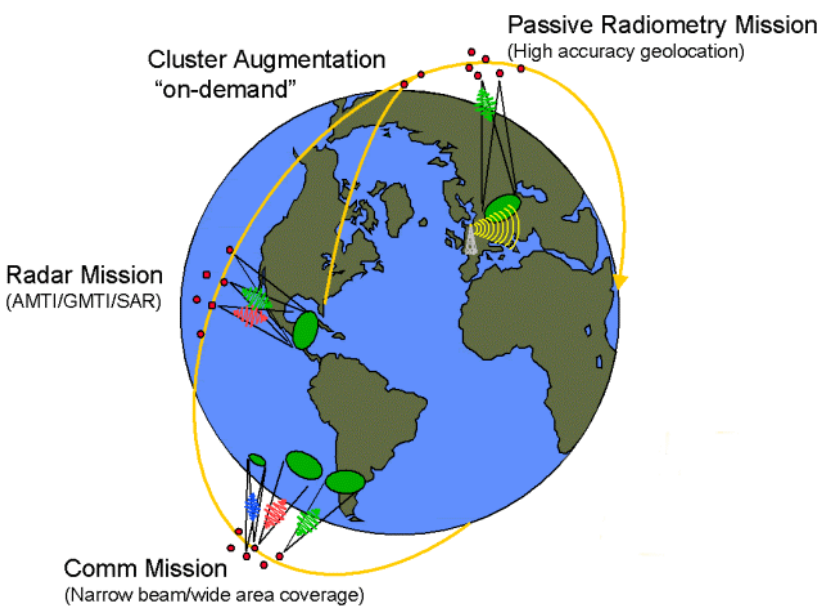

Figure 1 TechSat 21 Mission Concept (image from TechSat 21 Program Overview (AFRL 1998)).

The TechSat 21 program (Technology Satellite of the 21st Century) is a coordinated effort of several Air Force Research Laboratories directorates to study a variety of application missions for the satellite cluster concept. The initial focus of the program is on Ground Moving Target In- 
dication (GMTI), Synthetic Aperture Radar (SAR) imaging, formation-flying, and on-board autonomy.

Each micro-satellite in the cluster transmits radar pulses that are orthogonal to those transmitted by every other satellite in the cluster, and each detects and coherently combines the returns from every satellite in the cluster. In this way, the micro-satellites in the cluster form a large but sparse coherent array, enabling collection of angle- and time-of-return data with an effective aperture equivalent to the separation between the micro-satellites.

The vision for the deployed system is a constellation of "virtual" satellites, with each virtual satellite being a cluster of micro-satellites. The numbers are still to be determined, but according to one published account (Martin and Stallard 1999) there would be 35 virtual satellites and 5 spare virtual satellites. Each cluster would contain eight micro-satellites flying within 250 meters of each other. The constellation size is motivated by a desire for fullearth coverage and/or continuous coverage in two theatres. The number and spacing of the micro-satellites in each cluster is motivated by performance requirements for the GMTI mission. Other proposals mention cluster sizes up to 16 micro-satellites, and other missions (such as passive geolocation) require larger separations (up to $5 \mathrm{~km}$ ).

A one-year technology demonstration flight is planned for launch in November 2004. This flight will consist of one cluster of only three micro-satellites. This single cluster will reconfigure the orbits of its micro-satellites for different phases of its mission, which will test different mission applications.

This paper reports on an on-going project concerned with developing an automated planning assistance system for mission planning for the TechSat 21 flight experiment. Although this will experimental software developed through a research program, the intention is that it will be used operationally during the flight experiment.

\section{Payload Schedule Optimization}

The initial focus of the ground-based mission planner is optimization of the quantity and quality of observations that can be made during the entire mission life, taking into account detailed constraints regarding resource usage, viewing geometry, downlink opportunities and bandwidth, and interactions with bus activities. The planner creates a detailed schedule that attempts to maximize the number of target observations and the quality of the observations while distributing the observations as evenly as possible among all the identified targets according to preference.

\section{Target Selection}

Scheduling for any satellite whose mission involves observations of and/or communication with the ground must take into account "accesses"-windows of opportunity defined by when the satellite is in view of the target on the ground. These are determined by the precise orbit that the satellite is in. For satellite clusters, one must also take the relative positions of the individual satellites into account when assessing viewing geometry.

Satellites cannot fly side-by-side in close formation without expending a great deal of fuel. "Formation-flying" satellites actually fly in closely related stable orbits. Unless the satellites are flying directly behind one another, the only stable orbits involve relative elliptical motion. Thus, the geometric configuration of the satellites is in constant periodic flux. When an observation requires coordinated action by all satellites in the cluster, not only must all the satellites be in view of the target at the same time, but also their relative positions should satisfy the requirements for good observational geometry.

The mission planner must also use a performance predictor to decide which targets should be observed and when. There will often be several targets in a position to be observed at close to the same time. However, resource constraints will limit the number of targets that can be observed. For the demonstration flight especially, the objective will be to maximize both the number and quality of the observations made. This implies that the planner must select the targets to observe based on the predicted performance.

The performance predictor will be determined by a set of constraints specified by the payload experts for each type of observation. Go/no-go constraints will be used to filter out access opportunities for which there is little or no hope of a good observation. Figure of merit constraints will provide a means to compare the predicted observation quality for any two access opportunities.

\section{Ephemeris Uncertainty}

Spacecraft ephemeris will play a significant role in determining both exactly when target accesses are possible, and the figure of merit for the observation. Unfortunately, the spacecraft ephemeris cannot be predicted with the required degree of accuracy very far into the future.

This implies that the long-term plans made by the planner must be revisited and adjusted within a short interval (e.g., 72 hours or less) before each observation. Thus, one function of the mission planner will be to periodically compute updates to its plans as more accurate ephemeris data becomes available.

\section{Downlinks}

Synthetic aperture radar techniques generate very large amounts of data (the same is true for hyperspectral instruments). For example, the TechSat 21 micro-satellite 
(in a cluster with two other satellites) collects 9.6 Gigabytes (GB) per satellite for a two-minute observation. With overhead, this means that the total amount of data that must be downlinked from all three satellites is $33.5 \mathrm{~GB}$. At the expected downlink bandwidth of $150 \mathrm{Mbps}$, this will take approximately 32 minutes. In low earth orbit, the micro-satellites are in view of a ground station for approximately four minutes at a time, about four times a day. This means it can take two days to downlink the data from one two-minute observation (AFRL, 2000).

Thus, onboard memory capacity, downlink accesses and downlink bandwidth are the constraints that most limit the number of experiments that can be performed. The mission planner must select the ground station for each downlink taking care to observe constraints regarding contention for ground station resources.

\section{Downlink Uncertainty}

The situation is further complicated by the fact that whereas payload data will be downlinked via X-band communications with a commercial ground station, state of health downlinks and command uplinks will be communicated using S-band communications via the Air Force Satellite Control Network (AFSCN).

Thus, when payload data are downlinked, the memory they occupy onboard cannot be freed for reuse until the next contact opportunity with an AFSCN ground station, when the quality of the downlinked data can be verified. Not only are the X-band and S-band ground stations located in geographically distinct places, but there is a high degree of uncertainty regarding which AFSCN ground station will make the contact.

There are sufficient AFSCN ground stations that the satellites could be contacted several times a day. However, as an experiment, the TechSat 21 flight assumes a low priority in comparison with the operational satellite systems managed by AFSCN. The AFSCN performs a resource allocation determination on a daily basis. As a result, the TechSat 21 mission operations center will not know which potential contacts will actually occur until 24 hours before they happen.

This implies that the mission planner must allow for a high degree of uncertainty regarding when on-board resources will be released.

\section{Mission Operations Center}

The TechSat 21 flight experiment will be managed from a Mission Operations Center currently under development at AFRL Phillips Site in Kirtland AFB. All planning and scheduling functions will be coordinated through the Mission Planning Workstation (MPW) which will be running the Spacecraft Cluster Automatic
Planner/Scheduler (SpaceCAPS) software. Figure 2 shows the workstations with which the MPW will interface.

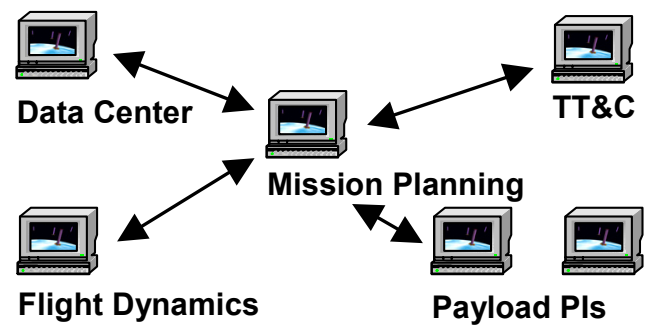

Figure 2. Mission Planning Workstation Interfaces

The Flight Dynamics workstation (FDW) will be used to track the space vehicles' orbits, perform orbit prediction, and plan both formation-keeping and formation-changing maneuvers. The MPW will obtain the ephemeris data it needs to perform its planning function by linking to a COTS orbit-propagation tool (Satellite ToolKit from Analytical Graphics, Inc.) running on that workstation. When the flight dynamics experts determine that a maneuver will be necessary, they will use the MPW's remote GUI to submit a demand that the maneuver be inserted into the schedule. Such demands will take precedence over observation requests.

The Telemetry, Tracking \& Commanding (TT\&C) workstation will take responsibility for processing the telemetry and tracking data obtained from the ground station, perform engineering analysis (including trending) on this data, and monitor the spacecraft state of health. It will also generate the command uploads to the spacecraft. The MPW will obtain state of health data affecting its planning and scheduling from the TT\&C workstation. It will submit its plans to the TT\&C workstation as a sequence of events, expressed as high-level procedure in the Satellite Control Language (SCL) from Interface \& Control Systems, Inc. The SCL system will expand the event sequence into the set of low-level satellite commands needed to carry out the plan, and submit these for upload to the ground station.

As the TT\&C workstation determines that housekeeping activities need to be executed, these will be submitted to the MPW for inclusion in the schedule.

All communication between the MPW and the TT\&C workstation will use the SCL message-passing protocol called the Software Bus

The payload experts will employ the MPW's remote GUI to submit requests to schedule observations. These requests will include specification of the payload constraints that determine viability and predicted value of each observation. The MPW will attempt to schedule as many of these observations as possible, subject to resource contention between the observations and between them and the formation-flying and housekeeping activities that 
are to be scheduled. The payload experts can use the MPW remote GUI to review the proposed schedules. It may not be possible to determine some payload configuration settings until the activity has been scheduled and the observation geometry has been determined. Furthermore, it may not be possible to automate the generation of these settings. Thus, the MPW remote GUI will also enable the payload experts to manually specify these settings before the schedule is submitted for uplink.

The Data Center is not technically part of the MOC. This workstation has a high-volume RAID disk system that will be used to provide organized storage of all the experiment data, telemetry data and configuration data. The Data Center will provide access to some of this data to offsite consumers via the internet. The MPW interfaces with the Data Center both to store the plans and schedules it creates, and to obtain current values for parameters affecting scheduling that characterize the spacecraft behavior, such as battery charge and discharge rates and slew times.

\section{SpaceCAPS Mission Planner}

The SpaceCAPS Mission Planner employs the Automated Scheduling and Planning Environment (ASPEN) system developed at JPL (Chien, 2000). SpaceCAPS encapsulates the ASPEN system within a Schedule Server that provides all the interfaces to the other workstations, including the Remote GUI.

The server performs some preprocessing of the activity requests (e.g., to use the payload go/no-go constraints to weed out potential observations). It also converts the emphemeris data obtained from the FDW into a sequence of activity requests for each access window. It provides heuristic functions to help control ASPEN's search process. It provides an access control mechanism that ensures that only authorized personnel can modify schedules and sanction them for upload to the satellites. Finally, it provides new way of viewing the schedules that emphasizes relationships between activities and the resources they employ.

\section{Status}

The SpaceCAPS mission planner is currently under development. It is now in Phase II of a Small Business Innnovative Research (SBIR) project.

We have demonstrated that we can obtain ephemeris and access window data from STK on the FDW via STK/Connect, obtain parameters from the Data Center and incorporate them into the ASPEN activity models, drive ASPEN to produce spacecraft schedules, convert the schedules to SCL scripts, archive the schedules in the Data Center, and communicate these scripts to the TT\&C workstation via the Software Bus for upload to the spacecraft.

Remaining efforts will concentrate on design and development of the Remote GUI, and improvement of the ASPEN activity models and search heuristics.

\section{Conclusions}

Formation-flying clusters of micro-satellites have several technical and economic advantages over monolithic satellites, but they also pose new technical challenges. Among these is the need for increased automation in mission planning and operations, and the need for planning and scheduling systems to be aware of new constraints and optimization criteria particular to cluster management. The SpaceCAPS project is identifying these new constraints and developing techniques to address them.

\section{Acknowledgements}

The author gratefully acknowledges the assistance provided by Charles Earl, Lance Self, Paul Zetocha, Ross Wainwrigth, Steve Fiedler, David Martin, and Maurice Martin. The work reported in this paper was supported by SBIR Phase II contract number F29601-01-C-0035 awarded by AFRL.

\section{References}

AFRL, 1998. TechSat 21 Program Overview. Air Force Research Laboratories Space Vehicles Directorate. http://www.vs.afrl.af.mil/VSD/TechSat21/. AFRL, 2000. TechSat 21 Command, Control and Communications Planning Document, V1.01 (Draft), May 2000. Chien, S., G. Rabideau, R. Knight, R. Sherwood, B. Engelhardt, D. Mutz, T Estlin, B. Smith, F. Fisher, T. Barrett, G. Stebbins, and D. Tran , "ASPEN - Automating Space Mission Operations using Automated Planning and Scheduling," SpaceOps 2000, Toulouse, France, June 2000.

Martin, M. and Stallard, M.J., 1999. Distributed Satellite Missions and Technologies — The TechSat 21 Program. In Proceedings of the 1999 AIAA Space Technology Conference and Exposition, Albuquerque, NM, 28-30 September 1999. AIAA-99-4479. 